\title{
EL ENFOQUE DE SISTEMAS MIGRATORIOS REVISITADO. UNA PROPUESTA TEÓRICA PARA EL ESTUDIO DEL SISTEMA MIGRATORIO DE AMÉRICA LATINA Y EL CARIBE
}

\author{
The Migration Systems Approach Revisited. \\ A Theoretical Proposal for the Study of the Migration System \\ of Latin America and the Caribbean
}

Marta Carballo de la Riva* Enara Echart Muñoz** María del Carmen Villarreal Villamar ${ }^{* * *}$

\begin{abstract}
Resumen. El objetivo de este artículo es revisitar los orígenes, desarrollo, límites y potencialidades del enfoque de sistemas migratorios, para formular una propuesta teórica que, a la hora de analizar la conformación, retroalimentación y reconfiguración de los sistemas migratorios, considere el papel de la estructura, de los factores de carácter meso (como las instituciones o los colectivos) y de la agencia migrante (individual y colectiva). Partimos para ello, de una perspectiva crítica dialéctica que establece un vínculo entre los elementos del sistema con los condicionantes estructurales, al tiempo que recupera la agencia migrante, principalmente en su dimensión colectiva. Nuestra propuesta será aplicada exploratoriamente al estudio del sistema migratorio de América Latina y el Caribe en el periodo 1970-2018. Más allá de una mera descripción de los principales rasgos del sistema y sus subsistemas, tratamos de mostrar los elementos que condicionan su estabilidad y evolución, resaltando, además de los elementos clásicos de los sistemas, el peso de los condicionantes estructurales, así como de las resistencias y estrategias desarrolladas por los colectivos migrantes.
\end{abstract}

Palabras clave: sistemas migratorios; América Latina y Caribe; estructura; agencia migrante.

\footnotetext{
Investigadora adscrita a la Unidad de Género de la Escuela de Gobierno (EG) y coordinadora académica del Máster en Género y Desarrollo (EG-Universidad Complutense de Madrid). Madrid, Espanha. Email: mcarball@ucm.es. Orcid: 0000-0003-4671-6891.

** Profesora de la Escuela de Ciencia Política de la Universidad Federal del Estado de Rio de Janeiro (UNIRIO) y coordinadora del Grupo de Investigación sobre Relaciones Internacionales y Sur Global (GRISUL). Rio de Janeiro - RJ, Brasil. Email: enara.munoz@unirio.br. Orcid: 0000-00020636-0301.

${ }^{* * *}$ Becaria Postdoctoral Nota 10 de FAPERJ en la Universidad Federal del Estado de Rio de Janeiro (UNIRIO) e investigadora del GRISUL y del NIEM. Rio de Janeiro - RJ, Brasil. Email: mariavillarreal85@gmail.com. Orcid:0000-0002-7255-2432.
} 


\begin{abstract}
The aim of this article is to revisit the origins, development, limits and potentialities of the migration systems approach. We will formulate a theoretical proposal that, when analyzing the conformation, feedback and reconfiguration of migratory systems, considers the role of the structure, of the meso factors (such as institutions or groups) and of the migrant agency (individual and collective). We start the analysis from a critical dialectical perspective that establishes a link among the elements of the system with its structural conditions, as well as recuperates the migrant agency, mainly in its collective dimension. Our proposal will be applied to the study of the migration system of Latin America and the Caribbean in the period 19702018. The proposal will go beyond a mere description of the main features of the system and its subsystems, to explain what are the elements that condition its stability and evolution. We will also highlight more than the classical elements of the system, the weight of structural conditions as well as the resistances and strategies developed by migrant collectives.
\end{abstract}

Keywords: Migration Systems; Latin America and the Caribbean; structure; migrant agency.

\title{
Introducción
}

La movilidad humana es una característica del momento actual que va a seguir perfilando la realidad social, económica, política, religiosa de las próximas décadas, lo que la sitúa como una de las cuestiones prevalentes en el mundo. Ser capaces de entender por qué las personas se mueven, qué dinámicas prevalecen y/o cambian y alteran la realidad móvil y cómo estas se relacionan con las estructuras y las propiedades que comprenden los sistemas migratorios, es el eje conductor de este trabajo. La interacción entre el comportamiento de los individuos y los contextos históricos, políticos, sociales y económicos ha originado patrones de interacciones relativamente estables que facilitan la migración posterior, entendidos como parte de un sistema migratorio (Fawcett, 1989; Bakewell, De Haas, Kubal, 2012; Bakewell, 2014).

El concepto de sistema migratorio ha sido utilizado a lo largo de las últimas décadas de forma aleatoria, bien como modelo teórico para analizar las migraciones, bien como elemento descriptivo para aludir al fenómeno sin profundizar en la propuesta teórica ni delimitar los elementos que subyacen al introducir la propia idea de sistema en el abordaje de la migración. En el presente artículo, serán objeto de nuestra atención las teorizaciones realizadas por diversos autores (Mabogunje, 1970; Kanaroglou, Liaw, Papageorgiou, 1986a; Fawcett, 1989; Portes, Boröcz, 1998; Kritz, Lim, Zlotnik, 1992; De Haas, 2010; Bakewell, De Haas, Kubal, 2012; Bakewell, 2014) para aproximarnos desde ellas y sus críticas a la concepción del sistema migratorio.

La diversidad de movimientos migratorios -locales, regionales, intrarregionales e internacionales-, las tipologías migratorias, los perfiles, 
los contextos en los que se imbrican los mismos y sus interacciones con los contextos global y glocal, orientan nuestra reflexión y estudio. En este sentido, el estudio de los patrones de movilidad humana y cambio social como procesos interconectados a partir de diversos factores (teorías de alcance medio, Castles, 2007, 2010; Portes, 1997, 2004; Massey et alii, 1993) se realizará integrando diversas aproximaciones teóricas que atiendan los contextos históricos y socioeconómicos en los que se insertan los sistemas migratorios.

La propuesta teórica de sistema migratorio permite entender el sistema como unidad analítica, integrando perspectivas más allá del estudio dicotómico origen/destino y analizando los numerosos elementos que interactúan dentro del mismo (Carballo, 2012). No obstante, creemos importante ampliar la capacidad explicativa de estos modelos incorporando una perspectiva crítica que nos permita complejizar los análisis. Así, entendemos que ese sistema está inserto en un contexto más amplio que debe ser considerado, ya que implica una serie de determinantes estructurales para la conformación de capacidades, instituciones e ideas, con incidencia en los ámbitos políticos, económicos y sociales (Cox, 1996). Ello no excluye la importancia de la agencia migrante en la medida en que, las estructuras pueden condicionar, pero no determinar la acción, que puede darse o no, en función de otros criterios (recursos, capacidad de movilización, existencia de una identidad colectiva, etc.), desafiando incluso esa estructura. Seguimos por tanto la tradición críticodialéctica ${ }^{1}$, que ofrece una superación del clásico debate entre agencia y estructura, mostrando las interacciones y tensiones entre ambas.

A nivel metodológico, nuestro trabajo está basado principalmente en la revisión de la literatura especializada sobre el enfoque de sistemas migratorios. Para formular nuestra propuesta teórica, dicha revisión ha sido complementada con el análisis estructural formulado por Wallerstein (1979) y Cox (1996), perspectivas autónomas para entender las especificidades de América Latina, como las desarrolladas por Quijano (2000) a propósito de la colonialidad del poder, y el análisis de datos secundarios para comprender algunos de los rasgos esenciales del sistema migratorio latinoamericano. En relación a la agencia migrante, inspiradas en la tradición crítico-dialéctica sobre la relación entre agencia y estructura, integramos también perspectivas que analizan la agencia colectiva de las y los migrantes (Carballo, Echart, 2012) y algunas formulaciones de exponentes del enfoque de la autonomía de las migraciones como Mezzadra (2012), De Genova, Mezzadra, Pickles (2015) y Varela (2015). Además, este trabajo es también fruto del análisis de documentos de organizaciones de migrantes y del seguimiento de sus redes sociales para conocer de cerca los

Hay numerosos autores representativos de esta perspectiva, tales como Castells, Giddens, Bourdieu o Habermas. Para un resumen de algunas teorías que integran la acción y la estructura, ver Ritzer (1993). 
principios y estrategias que orientan la acción colectiva de estos grupos. Si bien la pretensión de este artículo es abrir una discusión eminentemente teórica, como primer ejercicio práctico, exploraremos la capacidad explicativa del modelo analizando algunas de las dinámicas generales de la configuración del sistema migratorio de América Latina y el Caribe (ALC).

\section{La incidencia de la estructura en las migraciones}

Antes de adentrarse en el fenómeno especifico de las migraciones, es preciso entenderlas desde una perspectiva histórica en el contexto más amplio de transformaciones sociales en el que se originan, ya que existe una vinculación entre los sistemas migratorios y las estructuras en las que se enmarcan. En este sentido, partimos de las aportaciones de Wallerstein (1979) sobre el sistema mundo, un sistema histórico que se constituye en un espacio y tiempo específico, (desde el siglo XVI hasta la actualidad), y que articula una estructura económica (organizada alrededor de la acumulación de capital en el mercado mundial), política (Estados - Nación) y cultural, que lo legitima. Para él los sistemas poseen límites, estructuras, grupos, miembros, reglas de legitimación y coherencia, y tienen las características de un organismo (tiempo de vida a lo largo del cual permanecen estables y pueden modificarse).

Las ideas de Cox (1996) nos permiten analizar las estructuras históricas, definidas como configuraciones de fuerzas determinadas que condicionan la acción en un momento histórico concreto. Existen tres tipos de fuerzas que interactúan en una estructura:

- Las capacidades materiales, que generalmente se relacionan con el ámbito de la producción. Y aquí el lugar que los Estados y grupos sociales ocupan en el modo de producción es esencial para entender sus capacidades dentro de una determinada estructura.

- Las ideas, entendidas como significados intersubjetivos -nociones compartidas de la naturaleza de las relaciones sociales que tienden a perpetuar hábitos y expectativas de comportamiento, trascendiendo las divisiones sociales- y como imágenes colectivas -en torno a la naturaleza y legitimidad de las relaciones de poder existentes, de las nociones de justicia, etc.-. En el caso que nos ocupa, son los imaginarios colectivos en torno a las migraciones, entendidos la mayoría de las ocasiones como "problema" o "amenaza", que debe ser gestionado y/o solucionado, lo que facilita y justifica un determinado tipo de prácticas y políticas. Imaginarios que responden a los intereses de las potencias hegemónicas que ocupan un lugar central en el sistema mundo.

- Las instituciones, que permiten la estabilización y perpetuación de un orden determinado, reflejando las relaciones de poder establecidas, y potenciando imágenes colectivas acordes con esa estructura de poder, para legitimarlo. De este modo, es esencial tener en cuenta el papel de las organizaciones internacionales dedicadas a la elaboración de doctrina y políticas migratorias, en la actualidad englobadas en lo que definen como "gobernanza global de las migraciones". 
Para entender el funcionamiento de estas tres fuerzas, Cox (1987) adapta el concepto gramsciano de hegemonía, definido como:

cierto tipo de dominación en la que el estado dominante crea un orden basado ideológicamente en un amplio nivel de consenso, que funciona según los principios generales que aseguran de forma efectiva la supremacía continuada del estado o estados dominantes, pero al mismo tiempo ofrece cierto nivel de satisfacción al menos poderoso. (Cox, 1987, p. 7)

Cox aplicará esta noción en sus estudios sobre organizaciones internacionales, que, creadas por los Estados hegemónicos, permiten la expansión de sus valores y normas, que legitiman ideológicamente, y la creación de consenso en torno a ellos, mediante la cooptación de las elites de los países periféricos y la absorción de ideas contrahegemónicas para hacerlas consistentes con la doctrina oficial. El caso de la gobernanza global de las migraciones es un ejemplo de este tipo de consensos, que legitiman y afianzan una visión hegemónica del fenómeno migratorio, y que ha sido adoptada por diversos actores de una forma relativamente acrítica (Geiger, Pécoud, 2013; Domenech, 2017). Cox muestra la interacción entre agente y estructura en sus aproximaciones a las instituciones, que han sido socialmente construidas, como respuestas colectivas a problemáticas percibidas socialmente, y que producen determinadas prácticas. Esas mismas prácticas pueden verse desafiadas por nuevas ideas y acciones, que, en función de su alcance, podrán constituirse en fuerzas contrahegemónicas o ser cooptadas para la manutención del sistema hegemónico.

Si bien el concepto de estructura histórica se aplica al contexto más amplio de transformaciones sociales globales, sin duda el campo de las migraciones es paradigmático para entender cómo las capacidades materiales, las ideas y las instituciones actualmente existentes contribuyen para la legitimación y manutención de determinadas visiones y políticas migratorias al tiempo que dificultan o excluyen otras. El lugar de América Latina en esa estructura histórica, por tanto, va a tener una fuerte incidencia, como veremos, en la configuración de sus sistemas migratorios.

En este sentido, conviene tener presente también la idea de colonialidad del poder desarrollada por Quijano (2000). En su concepción de la colonialidad, el poder quedaría definido como una malla de relaciones sociales de explotación/dominación/conflicto que se articulan en función y en torno de la disputa por el control del trabajo y sus productos; la "naturaleza" y sus recursos de producción; el sexo, sus productos y la reproducción de la especie; la subjetividad y sus productos, incluido el conocimiento y la autoridad y sus instrumentos, que sirven para asegurar la reproducción del patrón de relaciones sociales. Estos elementos inciden en la configuración del sistema migratorio, imponiendo condicionantes estructurales a los diversos actores 
en función de su posicionamiento en las relaciones de poder hegemónicas, pero también apuntan caminos para su contestación, como se verá al incluir la agencia migrante de aquellos colectivos tradicionalmente excluidos.

El capitalismo sería la estructura mundial de poder en cuyo interior se articulan el trabajo y sus formas de explotación y control, siendo la colonialidad uno de los elementos principales del patrón mundial de poder capitalista (Quijano, Wallerstein, 1992). Este patrón de poder opera en diversos planos, ámbitos y dimensiones sociales, y se constituye a partir de la imposición de una clasificación racial/étnica de la población del mundo. El concepto complementa las propuestas teóricas de Wallerstein y Cox, y es necesario para entender las articulaciones de las relaciones sociales y las jerarquías en los países que constituyen un sistema migratorio (países centrales y periféricos en función de su posición en la división internacional del trabajo). Para el caso de ALC, desde la expansión del sistema colonial, la dinámica del sistema migratorio tendría características estables, lo que explicaría su pervivencia en el tiempo y espacio. Partiendo de estas características estructurales, podemos adentrarnos ahora en las propuestas teóricas de sistema migratorio.

\section{El enfoque teórico de sistema migratorio}

La noción de sistema ha sido ampliamente estudiada y utilizada por las ciencias sociales, sirviendo como fórmula proposicional para problematizar el mundo social (Pareto, 1935; Bertalanffy, 1950; Parsons, 1951; Bertalanffy, Ross Ashby, Weinberg, 1978; Luhmann, 1995; Pickel, 2007). Desde las aportaciones sobre sistemas, y más concretamente sobre sistema social, emerge el concepto de sistema migratorio.

Entendiendo, de manera general, un sistema como un conjunto de elementos con relaciones entre sí y con el medio ambiente, la primera propuesta que aparece asociada a las migraciones vendrá realizada por el sociólogo Mabogunje (1970). Éste, en base a las aportaciones de Bertalanffy ${ }^{2}$ (1950) en su teoría general de sistemas, aplica esta noción a la migración rural-urbana dentro del continente africano para tratar de explicar el tránsito de un migrante rural a un habitante urbano permanente (De Haas, 2010; Bakewell, 2014).

Un sistema puede definirse como un complejo de elementos que interactúan, junto con sus atributos y relaciones. Una de las tareas principales en la

2 En su teoría general del sistema, plantea una nueva doctrina científica de la "totalidad" e introduce como la característica básica de todo sistema orgánico el estado de cambio perpetuo de sus componentes. Este cambio en los componentes habla de entradas y salidas -sistema abierto-, constituyendo una especificidad de los organismos vivos, a diferencia de los sistemas cerrados donde la composición permanece constante. Los sistemas abiertos pueden llegar a un estado estacionario a partir de la constancia, que se alcanza con un cambio continuo en la entrada y salida de componentes. Para Bertalanffy los sistemas vivos son los ejemplos más importantes de sistemas abiertos y estados estables. 
conceptualización de un fenómeno como un sistema, por lo tanto, es identificar los elementos básicos que interactúan, sus atributos y sus relaciones. Una vez hecho esto, pronto se hace evidente que el sistema no opera en un vacío sino en un entorno especial ... Un sistema con su entorno constituye el universo de los fenómenos que son de interés en un contexto dado. (Mabogunje, 1970, p. 3)

Las partes que conforman el sistema serían: los elementos - i) entorno ambiental -condiciones económicas, políticas, valores sociales y comunitarios, disponibilidad de transporte y comunicaciones-, ii) las personas migrantes, iii) los subsistemas de control, iv) los mecanismos de ajuste, v) los mecanismos de retroalimentación (positivos y negativos)-, los atributos y las relaciones, que interactúan en un entorno en cambio constante (sistema abierto) que recibe estímulos del medio. Mabogunje también identifica varias dimensiones -espacial (horizontal) y socioeconómica (vertical)- y centrando su atención en los/as migrantes, las instituciones (subsistemas) y en las relaciones sociales, económicas y de otro tipo (como los mecanismos de ajuste) que forman parte integral del proceso de transformación del sistema. Define subsistema de control como "aquel que supervisa el funcionamiento del sistema general y determina cuándo y cómo aumentar o disminuir la cantidad de flujo en el sistema" (Mabogunje, 1970, p. 5).

Sus aportaciones, que más adelante serán aplicadas a la migración internacional (Fawcett, 1989; Kritz, Lim, Zlotnik, 1992; Massey et alii, 1993), permiten el encaje de las propuestas transnacionales -que analizan los vínculos entre comunidades, familias y personas a través del espacio-, y reconocen la existencia del sistema en relación a un contexto amplio. La existencia de estas comunidades da lugar a flujos estables que refuerzan o alteran las dinámicas del sistema y crean subsistemas a su vez. El carácter estable de las interconexiones es lo que perfila lo que Mabogunje señala como "estructura geográfica identificable que persiste a través del espacio y el tiempo" (1970, p. 12).

Para Mabogunje será la dinámica del sistema la que cambia el patrón de movimiento a lo largo del tiempo, es decir que los mecanismos de retroalimentación y los cambios en el ambiente, más allá de las condiciones iniciales que inician el movimiento, serán los que incidan en el estado del sistema (en la escala y la dirección de los flujos).

La preocupación sobre la evolución del sistema migratorio, saber qué cambios son los que lo alteran, qué mecanismos de retroalimentación y desequilibrios inciden en éste, será abordada por Kanaroglou, Liaw, Papageorgiou (1986a, 1986b) en dos trabajos que combinan la teoría de sistemas migratorios con la teoría de la utilidad estocástica (que tiene en cuenta variables aleatorias). Considerando el sistema con un número de individuos distribuidos a lo largo de diversas áreas -regionales, interurbanas, intraurbanas-, los autores exploran las decisiones individuales (a partir del modelo de elección) 
y los cambios agregados uniendo los niveles macro y micro del sistema, y definen las características de un sistema autónomo/no autónomo (dinámico).

Fawcett (1989) define el sistema migratorio como dos o más países unidos por flujos y contraflujos de personas. Su modelo integra las perspectivas macro y micro, y establece categorías - i) relaciones entre estados; ii) conexiones de la cultura de masas; iii) redes familiares y personales; iv) actividades de la agencia migrante)- y tipos de vínculos (tangibles, regulatorios y relacionales) en los sistemas migratorios. En la misma línea Kritz, Lim y Zlotnik (1992) aplicaron el modelo de Mabogunje a la migración internacional, integrando varias escalas y enfoques teóricos.

Nijkamp y Voskuilen (1996), para explicar los flujos hacia Europa, amplían la propuesta de Mabogunje dando más peso a los factores históricos y sociales y a su incidencia en la existencia y el mantenimiento de los flujos migratorios. Señalan cuatro componentes en el sistema: i) condicionantes macroestructurales -medioambiente, calidad de vida, situación económica y política, población, transporte y comunicaciones-; ii) motivos para moverse -económicos, sociales, satisfacción residencial, redes familiares y de amistad-; iii) elección del destino -motivos individuales afectados por otros elementos (políticas de migración, redes sociales, condiciones económicas y vínculos históricos y culturales)-; iv) proceso de absorción en el país de destino (mecanismos reguladores y de control -vivienda, empleo, integración-) (King, Skeldon, Vullnetari, 2008, p. 21).

Partiendo de las ideas de Mabogunje e incorporando las aportaciones de la década de los 90, Bakewell (2014) analiza las aproximaciones teóricas en torno a los sistemas migratorios y elabora una crítica sobre un uso un poco delimitado y esquelético del concepto. Así, señala como el enfoque queda convertido en un resumen de flujos obviando el poder explicativo y el desarrollo del funcionamiento interno de la propuesta de Mabogunje. "Si bien la idea del sistema de migración puede haber sido adoptada con cierto entusiasmo, el intento de delimitarlo con hallazgos empíricos ha tendido a dar como resultado versiones truncadas del enfoque" (2014, p. 303). Bakewell analiza también otros conceptos vinculados a la migración internacional y contenidos en la propuesta de sistema migratorio -los sistemas de retroalimentación y la causalidad acumulativa (Myrdal 1979; Massey, 1990; Faist, 2004; Canales, 20153)-, incluyendo las aportaciones críticas de De Haas (2010) y Bakewell,

La causalidad circular y acumulativa será desarrollada en primera instancia por Myrdal (1979) para explicar el círculo de crecimiento/decrecimiento en el que se encuentran las regiones del mundo. La causación circular permite la integración de un mayor número de factores para explicar las causas del crecimiento/decrecimiento. Estos factores serán causa y efecto de otros, originando que un cambio o alteración en uno produzca un cambio en el sistema de forma general. Es decir, si los cambios son positivos dinamizarán un proceso de crecimiento y desarrollo y viceversa si son negativos (causación recíproca circular). Esta idea será recuperada parcialmente por Massey (1990) para explicar las migraciones como un proceso social que se autoalimenta a partir de 
De Haas y Kubal (2012), sobre las redes, los mecanismos de retroalimentación endógenos, y la propia idea de retroalimentación.

Para Bakewell (2014) la propuesta teórica de sistema migratorio centra la mirada en las partes que constituyen el sistema, es decir, el enfoque de sistemas exige el análisis de las áreas de origen y de destino; y analiza los vínculos dinámicos entre estas áreas y las decisiones y flujos de migración. El enfoque debe analizar también los factores intermedios (como instituciones y políticas migratorias) y las formas en que las experiencias de migración en un período pueden moldear las condiciones para futuros movimientos. Además, va más allá de las explicaciones individualistas de los modelos neoclásicos de elección racional, al estudiar las relaciones dinámicas entre origen y destino y entre los diferentes niveles del análisis social, tratando de vincular la acción de los migrantes individuales y los cambios en los sistemas más amplios -estructura y agencia-. La propuesta de Bakewell complementa a Mabogunje, ampliando los límites del entorno (objetos o factores para los cuales los mecanismos de retroalimentación no pueden identificarse, por ejemplo, factores ambientales, enfermedades, conflictos políticos, tecnología, crisis económicas) y de la capacidad que éste tiene y viceversa, en el movimiento de las personas y en sus estrategias. Por tanto, en su propuesta señala la necesidad de definir las dinámicas del sistema (multicapa), especificar los mecanismos de retroalimentación (internos) y las relaciones entre los cambios en los elementos del sistema y el entorno.

\section{La agencia migrante}

Analizar la relación entre acción y estructura constituye, según Margaret Archer (1988), el problema central de la teoría social. El problema agenteestructura tiene dos dimensiones: una ontológica y otra epistemológica. La primera tiene que ver con qué son los agentes y las estructuras sociales y cómo se relacionan entre sí, mientras que la segunda se refiere al tipo de explicación utilizada para dar cuenta de ambas entidades. Esta última debe destacar las propiedades causales consideradas más relevantes, así como evidenciar si tienen mayor poder explicativo las interpretaciones que dan más peso a los agentes o, por el contrario, aquellas que se centran en las

ciertos mecanismos socioeconómicos (redes sociales, familiares, relaciones de solidaridad, cultura de emigración, etc.) que posibilitan su reproducción. Canales (2015), en sus estudios sobre la migración internacional también utiliza las tesis de Myrdal para vincularla a los procesos de reproducción social y de estructuras de desigualdad. Es lo que él define como reproducción en el ámbito global de una estructura en la que identifica grupos, estratos y clases sociales diversas pero articuladas e integradas en su reproducción social. Las ideas de Canales recuperan la estructura y las articulaciones locales-globales para entender la migración internacional como un componente de la reproducción del propio sistema mundo, “... a través de la migración se reproduce una estructura de desigualdad social ...con una forma transnacional" (Canales, 2015, p. 38). 
estructuras. Bien sea para considerarlos como factores independientes o para defender su integración, la relación entre agente y estructura ha sido objeto de interés de diversas disciplinas y perspectivas teóricas: expresándose como la relación entre factores micro y macroestructurales en la tradición norteamericana, o como el vínculo entre agente y estructura en la literatura de matriz europea (Ritzer, 1993).

En relación a los sistemas migratorios, los trabajos de Bakewell, De Haas y Kubal (2012), De Haas (2014) intentan complementar los análisis centrados en los factores macro, contextuales y estructurales para explicar el surgimiento de los sistemas de migración, con una teorización de la agencia de los migrantes pioneros. Aunque no resuelven la tensión entre estructura y agencia, exploran aspectos novedosos como las interacciones entre la agencia de los migrantes, el capital social "positivo y negativo", y el surgimiento de cualidades sistémicas particulares de los movimientos migratorios. En base a las aportaciones de Emirbayer y Mische (1998) sobre la agencia como categoría analítica, con sus tres niveles -interacional (habitual), proyectivo y práctico-evaluativo-, tratan de explicar las estrategias divergentes de los migrantes pioneros y su papel en alentar o desalentar la migración posterior y la formación y desintegración del sistema de migración.

Morawska (2007), por su parte, define la agencia como "el compromiso cotidiano de individuos con diferentes entornos estructurales que, a través de la interacción del hábito, la imaginación y el juicio, reproducen y transforman esas estructuras en respuesta interactiva a los problemas planteados por situaciones desafiantes" (Morawska, 2007, p. 12). Para la autora, la agencia del individuo estará supeditada por sus recursos socioculturales y por la influencia de varios elementos estructurales que varían espacial y temporalmente -el dinamismo o estancamiento de la economía; la naturaleza fluida o segmentada del mercado laboral y su grado de regulación; la apertura y el carácter restrictivo de las políticas estatales para la migración; y el pluralismo cívico-político o la exclusividad de la sociedad de acogida (Morawska, 2007, p. 13). En este sentido, también apunta De Haas (2014) que la agencia se ejerce dentro de las condiciones creadas por las estructuras, y que la capacidad real del individuo en el ejercicio de ésta y el cambio estructural que puede ejercer es limitado. La migración se entiende como un subproceso de procesos más amplios de transformación y desarrollo social, dentro de los cuales existe poco margen de maniobra.

Por el contrario, desde un enfoque crítico-dialéctico, autores como Anthony Giddens y Pierre Bourdieu ${ }^{4}$ desarrollaron perspectivas que integran

4 Otros enfoques que se inscriben en esta línea son los trabajos de Margaret Archer y Jürgen Habermas. Para una revisión detallada de las propuestas de estos y otros autores ver Ritzer (1993). 
la acción y la estructura. Así, mediante la teoría de la estructuración, o a través de la relación dialéctica entre habitus y campo, ambos teóricos mostraron que la constitución de los agentes y las estructuras no son independientes, sino que existe una relación dialéctica que guía y constriñe las acciones de los individuos, pero que también permite que, mediante sus luchas individuales o colectivas, éstos preserven o transformen las estructuras (Ritzer, 1993). En relación a los estudios migratorios, quienes se inscriben en esta línea de pensamiento, no ocultan los condicionamientos de las personas migrantes o la necesidad de entender los desplazamientos dentro de un contexto global específico, pero enfatizan la importancia de comprenderlos como agentes colectivos, considerándolos movimientos sociales (Carballo, Echart, 2012).

De forma complementar, el enfoque de la autonomía de las migraciones (Mezzadra, 2012; De Genova, Mezzadra, Pickles, 2015), además de cuestionar a los Estados, las fronteras, el régimen global de gestión de las migraciones o la misma noción de soberanía estatal, hace hincapié en la agencia individual, pero sobre todo colectiva de los y las migrantes. En particular, se subraya que estos (independientemente de su estatus migratorio), mediante sus prácticas, luchas y reivindicaciones, actúan de facto como ciudadanos/as, desafiando la noción tradicional de ciudadanía y permitiendo su ampliación y transformación (Mezzadra, 2012). Para Varela (2015, p. 156), la especificidad de estas luchas reside en que lo que moviliza a las personas es su condición de alteridad extranjerizada y no sólo su condición de trabajadores. Y es en este sentido en el que pueden suponer desafios a la hegemonia existente, abriendo con ello brechas en los consensos e imaginarios colectivos preestablecidos. No sin tensiones o contradicciones, en el caso de América Latina, algunos ejemplos de agencia colectiva migrante son las luchas y reivindicaciones a favor de la regularización y derechos, realizadas por migrantes en América del Sur, o las caravanas de migrantes en Centroamérica.

La propuesta teórica del sistema migratorio que elabora Mabogunje se verá enriquecida por las aportaciones y críticas posteriores, y nos valdremos de esta aproximación más incluyente para estudiar el sistema migratorio de ALC. Así, entendemos sistema migratorio como una estructura geográfica identificable que persiste a través del espacio y el tiempo (sistema abierto de larga duración), constituida por elementos en constante interacción en un entorno determinado. La existencia, pervivencia y alteración del sistema va a estar vinculada a numerosos mecanismos de retroalimentación existentes en espacios de carácter transnacional (vínculos dinámicos entre contextos de origen y destino, decisiones, flujos de migración, instituciones, estrategias y políticas migratorias, experiencias migratorias, etc.), y a las relaciones entre estructura y agencia. 


\section{Figura 1 - Elementos del sistema migratorio}

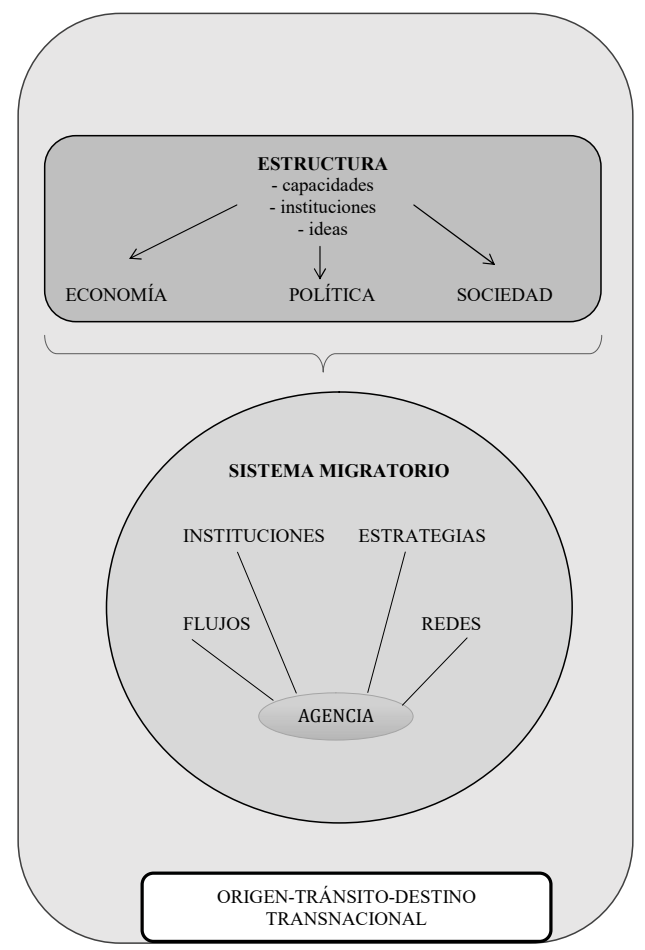

Fuente: Elaboración propia.

\section{El sistema migratorio en América Latina y el Caribe}

En América Latina y el Caribe la movilidad humana es una constante y constituye el correlato de la inserción internacional de la región en el sistema capitalista global. A partir del siglo XVI, los desplazamientos de carácter intrarregional y extrarregional fueron configurando una geografía migratoria de interacciones estables. Así, el sistema migratorio regional puede ser definido como una estructura geográfica basada en intercambios de información, cultura, bienes, capitales y desplazamientos de personas. Tal estructura permanece a lo largo del tiempo y del espacio e involucra a los países de la región, así como extrarregionales, conectados por vínculos históricos y contemporáneos de carácter político, económico o social. Dada la amplia heterogeneidad y perfiles migratorios (trabajadores inmigrantes, refugiados/as, turistas, estudiantes, artistas, etc.) de los países de ALC, el sistema migratorio regional está dividido en diversos subsistemas que obedecen a una división por subregiones: México y América Central, Caribe, y región andina y Cono Sur en América del Sur (Martínez, Cano, Soffia, 2014; Martínez, Orrego, 2016; Mejía, 2018; Canales, Rojas, 2018; Stefoni, 2018). 
A partir de los elementos señalados en nuestra propuesta teórica, en la configuración y transformaciones del sistema migratorio de ALC interactúan tres clases de elementos que veremos a continuación: factores de carácter estructural (sistema económico, relaciones internacionales, medio ambiente), junto a factores intermedios (instituciones, políticas migratorias) y la agencia migrante (individual o colectiva).

Respecto a la estructura, según los planteamientos antes expuestos de Cox (1996), para entender el sistema migratorio de ALC es preciso considerar la posición periférica y dependiente que la región ha ocupado históricamente, y sigue ocupando, en la división internacional del trabajo definida por el sistema mundo. Asimismo, es imprescindible rescatar la noción de colonialidad del poder de Quijano (2000) que caracteriza el patrón de dominación global propio del sistema mundo capitalista que surge con el colonialismo europeo desde el siglo XVI. Si analizamos históricamente las migraciones en ALC, veremos que las migraciones modernas están íntimamente vinculadas a la posición que la región ocupa a nivel internacional y responden a desigualdades internacionales, a las necesidades de mano de obra de los mercados y a los regímenes de control de la movilidad internacional. Así, en el contexto de inserción de ALC a la economía mundo, la primera fase migratoria de la región se extendió a lo largo de los siglos XV y XIX, y estuvo marcada por la adopción del extractivismo y la consecuente especialización en la producción de materias primas. En este marco, se promovió la llegada de población europea con fines de población y administración de las colonias, mientras que, para suplir las necesidades de mano de obra local, se organizó la llegada de población africana y asiática (especialmente de China e India), en condiciones de esclavitud y semiesclavitud (Pellegrino, 2003).

En la segunda fase migratoria regional (finales del siglo XIX-inicios del XX), la llegada masiva, sobre todo de población europea ${ }^{5}$ (Pellegrino, 2003; Martínez, Orrego, 2016) fue provocada por el hambre, la pobreza, las guerras o la búsqueda de una vida mejor, pero también fue alentada por las demandas de mano de obra de las economías latinoamericanas y caribeñas, entonces en plena expansión. En el proceso de consolidación de los Estados nacionales, la llegada de esta población fue también resultado de políticas selectivas de atracción impulsadas por visiones ideológicas que buscaban promover el "blanqueamiento", el "progreso" o la "civilización" de las sociedades regionales, mediante la llegada de población "blanca" y considerada "superior" (Acosta, 2018).

Múltiples causas en los contextos de origen, las mejores condiciones de ALC respecto a otras áreas y sus políticas migratorias, también permitieron que colectivos "menos deseados" como japoneses, chinos, árabes y judíos ingresaran en esta época, aunque en proporciones mucho menores. 
A partir de la primera mitad del siglo XX empieza la tercera fase migratoria que se extiende hasta mediados de la década del sesenta (Pellegrino, 2003), caracterizada por la consolidación del modelo primario-exportador y, tras la Segunda Guerra Mundial, por la implementación de la estrategia de Industrialización por Sustitución de Importaciones. Como corolario, se experimentó un crecimiento demográfico y económico, así como procesos de modernización y urbanización. En función del modelo económico hegemónico y de las necesidades de mano de obra de las economías locales, se dieron amplias migraciones internas del campo a las ciudades, pero también intrarregionales y, como consecuencia de la represión de gobiernos militares, migraciones forzadas. Otro rasgo predominante de esta etapa es la emigración, mayoritariamente hacia los Estados Unidos (Zavala, Prieto, 2014).

La cuarta fase migratoria de la región empieza a finales del siglo XX (Pellegrino, 2003) y consolida a América Latina como emisora de mano de obra. En este periodo la región sigue recibiendo inmigrantes europeos, pero serán las migraciones Sur-Sur, con una importante presencia de migrantes intrarregionales y de africanos y asiáticos, las que más crecen (Martínez, Orrego, 2016). Estos procesos son resultado, por un lado, del endurecimiento de las políticas migratorias en los países del Norte y de la crisis económica internacional de 2008, pero también del crecimiento económico de la región, experimentado a inicios del siglo XXI durante el boom de las commodities, con mejores indicadores sociales, demanda de mano de obra y estabilidad política.

Desde 1970, ALC es una región prevalentemente de emigración y hoy constituye una de las áreas de emisión más importantes del mundo. De acuerdo con la última ronda censal, en 2010 existían 28.5 millones de personas residiendo fuera de la región, especialmente en Estados Unidos, Europa y Japón. Aunque la media regional de la emigración es del 4\%, existen importantes variaciones subregionales $-11,1 \%$ en el Caribe y $10,2 \%$ en Centroamérica, frente al 1,6\% de América del Sur- (Martínez, Cano, Soffia, 2014, p. 13).

Los condicionantes de la salida de población en esta fase pueden ser explicados en buena medida a partir de dinámicas estructurales. Tras el fin de la estrategia de industrialización por sustitución de importaciones, en los ochenta la región vive la "década perdida" y la implementación de programas económicos neoliberales que generan severos impactos sociales, ahondando la pobreza, las desigualdades y la dependencia con relación a los países del Norte que la caracterizan históricamente. Así, la evolución del modelo de desarrollo orientará buena parte de los flujos regionales de emigración. En el ámbito político, ALC vive una convulsionada fase de inestabilidad y conflictos civiles o sociales que también contribuyen a la salida de población (Pellegrino, 2003). En décadas más recientes y en pleno proceso de reprimarización de las economías de la región, a la emigración económica, de exilio y refugio, hay 
que agregar también la migración motivada por razones ambientales como los frecuentes huracanes, fuertes lluvias o sequías que azotan a la región, así como la derivada de la violencia generalizada que impera a raíz de la actuación de redes del crimen organizado (Martínez, Orrego, 2016; Canales, Rojas, 2018).

Como ilustra la figura 1 de nuestra propuesta teórica, además de los factores estructurales, para comprender el sistema migratorio de ALC, es preciso considerar el papel que desempeñan factores intermedios como las instituciones, las políticas o las redes migratorias. Estos elementos son los responsables por la permanencia y reajustes del sistema a través de los mecanismos de retroalimentación que generan. Al margen de las desigualdades o los vínculos históricos entre países, si analizamos, por ejemplo, la migración histórica de centroamericanos hacia los Estados Unidos, veremos que, en buena medida, la continuidad de los flujos migratorios se explica por la acción de las redes de migrantes. Estas últimas son un factor clave en el desarrollo de procesos migratorios y cumplen un papel esencial en la decisión de emigrar, en la selección de los destinos o periodicidad de la migración, aun cuando las causas que alimentan este proceso desaparezcan. Así, los Estados Unidos son el destino histórico de la migración centroamericana, y hoy concentran el $88,9 \%$ de los emigrantes salvadoreños, $86,6 \%$ de los guatemaltecos y $81,8 \%$ de los hondureños (Canales, Rojas, 2018, p. 14).

Además de las redes, la acción de factores intermedios como las instituciones o las políticas migratorias no sólo explican los elementos de continuidad en un sistema migratorio, sino también sus rupturas y mecanismos de reajuste como el aumento migración intrarregional en ALC. Es decir, aunque las migraciones de personas oriundas de la región son un proceso histórico, el desarrollo y consolidación de este fenómeno como una de las principales características del perfil migratorio regional es resultado de los mecanismos de retroalimentación que han generado las instituciones y políticas migratorias regionales. En efecto, si en 1970 la migración intrarregional suponía el 24\% del total, en 2010 equivalía al 63\% (Martínez, Orrego, 2016). El aumento de las migraciones intrarregionales ha sido especialmente evidente en América del Sur, donde más del 70\% de los migrantes es originario de la región. En este proceso fueron centrales la adopción de políticas migratorias relativamente liberales, así como la firma de Acuerdos de libre circulación o residencia, especialmente en el marco de procesos de integración regional como la Comunidad Andina (CAN), el MERCOSUR y la UNASUR (Villarreal, 2018; Acosta, 2018). A inicios del siglo XXI, tales acontecimientos tuvieron lugar en una coyuntura específica caracterizada por el crecimiento económico de las economías regionales, relativa estabilidad política y un alto nivel de convergencia ideológica entre gobiernos mayoritariamente progresistas. 
Los destinos históricos de la inmigración en América del Sur fueron tradicionalmente Argentina y Venezuela, pero a raíz de la crisis política, económica y social de Venezuela, esta última perdió su condición de país esencialmente receptor, para convertirse en el principal expulsor de población de la región -un éxodo calculado en más de cuatro millones de personas-, el $75 \%$ de los cuales se concentra en América del Sur. En la subregión, algunos de los destinos más importantes son aquellos países como Colombia o Ecuador que mantenían vínculos transnacionales previos, debido a la presencia significativa de migrantes colombianos o ecuatorianos en Venezuela (OIM, 2019). Con todo, países como Chile, Perú o Brasil se han posicionado también como importantes polos de atracción. En este contexto, las redes de migrantes y las políticas migratorias tienen un papel esencial en la selección de los destinos y en la reconfiguración de la posición de algunos países dentro del subsistema migratorio de América del Sur. Además, el ejemplo venezolano permite entender cómo las crisis coyunturales de carácter económico, político o social son también uno de los factores más importantes para comprender la salida masiva de población en cortos periodos de tiempo (Stefoni, 2018, p. 11).

La centralidad de la acción de factores intermedios también es visible en la emigración de los latinoamericanos y caribeños hacia Europa y países como Japón. Además de las asimetrías entre estas zonas y ALC, entre los principales factores que favorecen estos desplazamientos destacan las relaciones históricas de carácter colonial, políticas migratorias de atracción de mano de obra y acuerdos bilaterales que favorecen la inmigración a partir de criterios de etnicidad o proximidad lingüística, especialmente en los casos de España, Italia y Portugal (Durand, Massey, 2010, p. 38). A estos elementos, cabe agregar el papel de los vínculos transnacionales y de las redes migratorias en la continuidad de los desplazamientos más allá de sus causas.

Junto al papel que juegan la estructura y los factores intermedios, nuestra propuesta teórica defiende la necesidad de considerar el rol que desempeña en un sistema migratorio la agencia migrante. Como hemos visto, este elemento ha sido objeto de interés de autores que analizaron la agencia de los migrantes pioneros en la conformación de sistemas migratorios. Sin embargo, nuestro modelo va más allá. Además de la agencia individual, consideramos la agencia colectiva de los/las migrantes que, entendidos como movimientos sociales, tienen la capacidad de cuestionar y erosionar, aunque sea parcialmente, las estructuras en las que se verifican los procesos migratorios y los imaginarios que las sostienen. En el caso que nos ocupa, un ejemplo emblemático de la agencia migrante comunitaria son las caravanas migrantes registradas hacia los Estados Unidos desde octubre de 2018.

Aunque las caravanas migrantes son formas de movilización forzada a consecuencia de factores estructurales como la pobreza, la violencia 
generalizada, la inseguridad o la crisis ambiental que afecta a los países centroamericanos, en sus tres diversas "oleadas" fueron utilizadas como recurso para generar autoprotección de los/as migrantes frente a los peligros de la migración y las acciones del crimen organizado, obtener visibilidad mediática y posibilidades de protección internacional (Gandini, 2019). No obstante, expresaron también una forma de organización colectiva, con amplia participación de familias, que denunció, por un lado, una crisis humanitaria sin precedentes en los países del Triángulo Norte y, por otro, supuso una reivindicación del derecho a la sobrevivencia, a la libre movilidad y a gozar de una vida plena ${ }^{6}$. A título ilustrativo podemos destacar la movilización de activistas y de colectivos de migrantes autoconvocando ${ }^{7} \mathrm{u}$ organizando caravanas a través de Facebook y grupos de WhatsApp ${ }^{8}$, lo que denota capacidad de agencia en un escenario no exento de contradicciones y condicionantes.

Aunque las caravanas no son una novedad en el panorama migratorio mexicano, las registradas desde 2018 tuvieron lugar en un contexto de cambios en las políticas migratorias del gobierno mexicano y recibieron respuestas diferenciadas según el momento político del país (Narváez, 2019). Desde su inicio han estado sometidas a diversas estrategias de violencia, control y violación de los derechos humanos de los/as migrantes, pero bien sea por el volumen, visibilidad de sus demandas y sobre todo por la capacidad organizativa demostrada, constituyen un fenómeno sin precedentes en las migraciones contemporáneas. En este escenario, la movilidad se convierte en un elemento afirmativo y reivindicativo que busca dignificar las condiciones de vida de los/as migrantes. Las personas migrantes dejan así de ser solamente una consecuencia de los desequilibrios estructurales para convertirse en sujetos políticos capaces de organizarse y articular demandas concretas que cuestionan las estrategias

6 Para visibilizar las razones que motivaron su migración forzada, pero también los objetivos de sus proyectos migratorios, algunos ejemplos de las expresiones y lemas más utilizados en estos espacios fueron: "Emigramos por un futuro mejor", "no nos vamos porque queremos, nos expulsa la violencia y la pobreza", "abandonamos un país corrupto y en decadencia que no nos ofrece nada", "nos vamos porque no aguantamos el hambre y la violencia", "buscamos refugio, en Honduras nos matan" (informaciones recabadas en las redes sociales Facebook y Twitter de colectivos y organismos que participaron en las caravanas).

7 Aunque las caravanas fueron autoconvocadas por organizaciones de migrantes, se ha señalado que la alta repercusión que alcanzaron fue resultado de la amplia cobertura que realizaron medios de comunicación locales. De forma semejante, se considera que otro elemento útil para el crecimiento de las caravanas fue la publicación de sus comunicados en las redes sociales de políticos como Bartolo Fuentes, periodista y ex diputado de oposición en Honduras.

8 Pese a que los grupos de WhatsApp son generalmente cerrados, diversos medios de comunicación han tenido acceso a estos espacios y publicado periódicamente partes de diálogos. Un ejemplo es el del Diario Expediente Público que publicó algunos de los mensajes intercambiados por migrantes hondureños que participaban en una caravana. Fuente: <http://expedientepublico. org/un-dia-en-el-whatsapp-de-los-migrantes-hondurenos/>. 
tradicionales de control, gestión e interpretación del fenómeno migratorio, así como nociones clásicas sobre Estados, fronteras o ciudadanía.

También en el caso de América del Sur, los avances obtenidos a principios del siglo XXI sobre libre movilidad y el reconocimiento de derechos que han facilitado los desplazamientos en las últimas décadas no se comprenden si no analizamos el papel de la agencia colectiva de la población migrante. Sin soslayar la importancia de la estructura y la coyuntura en las que fueron concebidas, tales políticas son también fruto del activismo y de las luchas colectivas de migrantes en la subregión, como muestran el activismo a favor de medidas migratorias con enfoque de derechos humanos, las reivindicaciones de regularización y papeles para todos, la organización de huelgas o protestas migrantes y la concepción de campañas regionales para obtener derechos de ciudadanía 9 .

\section{Conclusiones}

Nuestro objetivo en este artículo ha sido debatir algunos enfoques de sistemas migratorios para tratar de presentar un modelo que integre los diversos elementos que los componen, al tiempo que los relaciona con aspectos estructurales y con la agencia migrante, entendiendo que su conformación, consolidación y cambio se da en función de multiples variables interrelacionadas. Pretendemos con ello abrir un debate que permita mejorar la capacidad explicativa de estos enfoques, atendiendo a su aplicabilidad en diversos escenarios, lo que sin duda constituye una agenda de investigación a futuro.

A modo de ejemplo exploratorio, se ha presentado el sistema migratorio de ALC a partir del modelo propuesto. Las fases migratorias de ALC dan cuenta de un sistema migratorio regional, caracterizado por diversos subsistemas, con rasgos diferenciados y elementos que condicionan su estabilidad y evolución. En este escenario, hemos visto que la emigración extrarregional e intrarregional (de carácter voluntario, forzado y mixto) es el patrón dominante, pero la inmigración de personas también se mantiene como un rasgo característico, actualmente sobre todo en dirección Sur-Sur.

Con una mirada de largo plazo, vimos también cómo, desde el siglo XVI, las desigualdades estructurales que caracterizan la economía mundial y la posición subordinada que la región ocupa en el sistema mundo han marcado los orígenes del sistema migratorio regional. A su vez, el análisis de las diversas etapas migratorias nos ha permitido entender las líneas de continuidad y las amplias reconfiguraciones que este sistema (y sus varios subsistemas) han experimentado de forma progresiva. De acuerdo con nuestra propuesta teórica,

9 Como ejemplo, las movilizaciones de las organizaciones de migrantes para la aprobación de medidas y legislaciones con enfoque de derechos en Argentina o Ecuador, o la campaña regional permanente "Aquí Vivo, aquí voto" que reivindica el derecho de voto para migrantes. 
tales procesos deben ser analizados a la luz de tres elementos. En primer lugar, se deben considerar los factores estructurales como el sistema capitalista, las desigualdades estructurales de la economía mundo, la colonialidad del poder, y los modelos de desarrollo regionales, cuyos efectos sociales y ambientales empujan a cada vez más personas a abandonar sus lugares de origen. En segundo lugar, deben ser incluidos elementos intermedios como las políticas, las instituciones o las redes que condicionan y alimentan los desplazamientos migratorios. Por último, la evolución de los sistemas migratorios no puede desconsiderar el papel contestatario y en ocasiones contrahegemónico que desempeña la agencia de los migrantes. Más allá de los estudios tradicionales sobre la agencia individual de los migrantes, nuestra propuesta tripartita incorpora el papel de las luchas y reivindicaciones colectivas de migrantes.

Con este trabajo, no hemos pretendido un análisis exhaustivo del sistema migratorio latinoamericano, sino apuntar una serie de elementos que permitan entenderlo y estudiarlo a posteriori, abriendo con ello una agenda de investigación que contribuya a profundizar en esos elementos y sus interacciones, así como otros contextos y realizar análisis comparados, superando algunos de los límites de los enfoques clásicos de sistemas migratorios.

\section{Referencias}

ACOSTA, Diego. The National versus the Foreigner in South America. 200 Years of Migration and Citizenship Law. Cambridge: Cambridge University Press, 2018.

ARCHER, Margaret. Culture and Agency: The Place of Culture in Social Theory. Cambridge: Cambridge University Press, 1988.

BERTALANFFY, Ludwig von. An Outline of General System Theory. British Journal for the Philosophy of Science, v. 1, n. 2, p. 139-164, 1950.

BERTALANFFY, Ludwig von; ROSS ASHBY, William; WEINBERG, Gerald M. Tendencias en la teoría general de los sistemas. Madrid: Alianza Editorial, 1978.

BAKEWELL, Oliver. Relaunching Migration Systems. Migration Studies, v. 2, n. 3, p. 300-318, 2014.

BAKEWELL, Oliver; DE HAAS, Hein; KUBAL, Agnieska. Migration systems, pioneers and the role of agency. Journal of Critical Realism, v. 11, n. 4, p. 413-437, 2012.

CANALES, Alejandro I. E Pur Si Muove. Elementos para una teoría de las migraciones en el capitalismo global. Guadalajara: Universidad de Guadalajara, 2015.

CANALES, Alejandro; ROJAS, Martha. Panorama de la migración internacional en México y Centroamérica. Serie Población y Desarrollo 124. Santiago: CEPAL, 2018.

CARBALLO, Marta; ECHART, Enara. Migrações e movimentos sociais: transnacionalismo, mobilidades e mobilizações. In: BRINGEL, Breno; GOHN Maria (orgs.). Para além do "novo": o estudo dos movimentos sociais na era global. Rio de Janeiro/Petrópolis: Editora Vozes, 2012, p. 147-174. 
CARBALLO, Marta. Movilidad intrarregional en América Latina: sistemas generizados. XV Encuentro de Latinoamericanistas Españoles, Nov 2012, Madrid, España, 2012, p. 1318-1336.

CASTLES, Stephen. Twenty-first century migration as a challenge to sociology. Journal of Ethnic and Migration Studies, v. 33, n. 3, p. 351-371, 2007.

CASTLES, Stephen. Understanding Global Migration: A Social Transformation Perspective. Journal of Ethnic and Migration Studies, v. 36, n. 10, p. 1565-1586, 2010.

COX, Robert. Social forces, states, and world order. In: COX, Robert; SINCLAIR, Timothy. Approaches to World Order. Cambridge: Cambridge University Press, 1996.

COX, Robert. Production, Power, and World Order: Social Forces in the Making of History. New York: Columbia University Press, 1987.

DE HAAS, Hein. The Internal Dynamics of Migration Processes: A Theoretical Inquiry. Journal of Ethnic and Migration Studies, v. 36, n. 10, p. 1587-1617, 2010.

DE HAAS, Hein. Migration theory: Quo vadis? IMI Working Paper No. 100 / DEMIG Project Paper No. 24. Oxford: International Migration Institute, 2014.

DE GENOVA, Nicholas; MEZZADRA, Sandro; PICKLES, John (eds.). New Keywords: Migration and Borders. Cultural Studies, v. 29, n. 1, p. 55-87, 2015.

DOMENECH, Eduardo. Las políticas de migración en Sudamérica: elementos para el análisis crítico del control migratorio y fronterizo. Revista Terceiro Milênio, v. 8, n. 1, p. 19-48, 2017.

DURAND, Jorge; MASSEY, Douglas. Nuevo orden mundial: continuidades y cambios en las migraciones latinoamericanas. In: DONATO, Katharine; HISKEY, Jonathan; DURAND, Jorge; MASSEY, Douglas S. (coords.). Salvando fronteras. Migración internacional en América Latina y el Caribe. México DF: Miguel Angel Porrúa, 2010, p. 19-49.

EMIRBAYER, Mustafa; MISCHE, Ann. What Is Agency? American Journal of Sociology, v. 103, p. 962-1023, 1998.

FAWCETT, James. Networks, Linkages, and Migration Systems. International Migration Review, v. 23, n. 3, p. 671-680, 1989.

FAIST Thomas. Towards a Political Sociology of Transnationalization. The State of the Art in Migration Research. European Journal of Sociology / Archives Européennes de Sociologie / Europäisches Archiv für Soziologie, v. 45, n. 3, p. 331-366, 2004.

GANDINI, Luciana. Las "oleadas" de las caravanas migrantes y las cambiantes respuestas gubernamentales. Retos para la política migratoria. In: FERNÁNDEZ, Alethia; GANDINI, Luciana; GUTIÉRREZ, Eduardo; NARVÁEZ, Juan. Caravanas migrantes: las respuestas de México. México: UNAM, 2019, p. 23-31.

GEIGER, Martin; PÉCOUD, Antoine (eds.) Disciplining the Transnational Mobility of People. Houndsmill/ Basingstoke: Palgrave McMillan, 2013.

KANAROGLOU, Pavlos; KAO, Liaw; PAPAGEORGIOU, Yorgos. An Analysis of Migratory Systems: 1. Theory. Environment and Planning A, v. 18, p. 913-928, 1986a. 
KANAROGLOU, Pavlos; KAO, Liaw; PAPAGEORGIOU, Yorgos. An Analysis of Migratory Systems: 2. Operational Framework. Environment and Planning A, v. 18, p. 1039-1060, 1986b.

KING, Russell. Theories and Typologies of Migration: An Overview and A Primer. Willy Brandt Series of Working Papers in International Migration and Ethnic Relations, n. 12, p. 1-43, 2012.

KING, Russell; SKELDON, Ronald; VULLNETARI, Julie. Internal and international migration: bridging the theoretical divide. Sussex Centre for Migration Research Working Papers, n. 52. Brighton, GB: University of Sussex, 2008.

KRITZ, Mary; LIM, Lea; ZLOTNIK, Hania (eds.). International Migration Systems: A Global Approach. Oxford: Clarendon Press, 1992.

LUHMANN, Niklas. Social Systems. Stanford, CA: Stanford University Press, 1995.

MABOGUNJE, Akin. Systems Approach to a Theory of Rural-Urban Migration. Geographical Analysis, v. 2, n. 1, p. 1-18, 1970.

MANGALAM, Joseph J.; SCHWARZWELLER, Harry K. Some Theoretical Guidelines toward a Sociology of Migration. International Migration Review, v. 4, n. 2, p. 5-21, 1970.

MARTÍNEZ, Jorge; CANO, Verónica; SOFFIA, Magdalena. Tendencias y patrones de la migración latinoamericana y caribeña hacia 2010 y desafíos para una agenda regional. Serie Población y Desarrollo, n. 109. Santiago: CEPAL, 2014.

MARTÍNEZ, Jorge; ORREGO, Cristián. Nuevas tendencias y dinámicas migratorias en América Latina y el Caribe. Serie Población y Desarrollo, n. 114. Santiago: CEPAL, OIM, 2016.

MASSEY, Douglas. Social Structure, Household Strategies, and the Cumulative Causation of Migration. Population Index, v. 56, p. 3-26, 1990.

MASSEY, Douglas; ARANGO, Joaquín; GRAEME, Hugo; KOUAOUCl, Ali; PELLEGRINO, Adela; TAYLOR, J. Edward. Theories of International Migration: A Review and Appraisal. Population and Development Review, v. 19, n. 3, p. 431466, 1993.

MEJÍA, William. Panorama de la migración internacional en el Caribe. Serie Población y Desarrollo, n. 122, Santiago: CEPAL, 2018.

MEZZADRA, Sandro. Capitalismo, migraciones y luchas sociales. La mirada de la autonomía. Nueva Sociedad, n. 237, p. 159-178, 2012.

MORAWSKA, Eva. International Migration: Its Various Mechanisms and Different Theories that Try to Explain it. Willy Brandt Series of Working Papers in International Migration and Ethnic Relations, 1/07. Malmö: Malmö University, 2007.

MYRDAL, Gunnar. Teoría económica y regiones subdesarrolladas. México: Fondo de Cultura Económica, 1979.

NARVÁEZ, Juan. Hasta topar con pared. Historia reciente de la migración en tránsito por México. In: FERNÁNDEZ, Alethia; GANDINI, Luciana; GUTIÉRREZ, Eduardo; NARVÁEZ, Juan. Caravanas migrantes: las respuestas de México. Series Técnicas sobre Temas de relevancia Nacional, México: UNAM, n. 8, 2019, p. 11-21. 
NIJKAMP, Peter; VOSKUILEN, Maurice. International Migration: A Comprehensive Framework for a Survey of the Literature. European Spatial Research and Policy, v. 3, n. 1, p. 5-28, 1996.

Organización Internacional para las Migraciones (OIM). Tendencias migratorias en las Américas, julio de 2019. Buenos Aires: OIM, 2019.

PARETO, Vilfredo. The mind and society. Trattato di sociologia generale. New York: Harcourt, Brace and Company, 1935.

PARSONS, Talcott. The Social System. London: Routledge \& Kegan Paul, 1951.

PELLEGRINO, Adela. La migración internacional en América Latina y el Caribe: tendencias y perfiles de los migrantes. Serie Población y Desarrollo, n. 35. Santiago: CEPAL, 2003.

PICKEL, Andreas. Rethinking Systems Theory. Philosophy of the Social Sciences, v. 37, p. 391-407, 2007.

PORTES, Alejandro. Neoliberalism and the Sociology of Development: Emerging Trends and Unanticipated Facts. Population and Development Review, v. 23, p. 229-259, 1997.

PORTES, Alejandro. La sociología en el continente: convergencias pretéritas y una nueva agenda de alcance medio. Rev. Mex. Sociología, v. 66, n. 3, p. 447-483, 2004.

PORTES, Alejandro; BÖRÖZCCZ, József. Migración contemporánea. Perspectivas teóricas sobre sus determinantes y sus modalidades de incorporación. In: MALGESINI, Graciela (comp.). Cruzando fronteras: migraciones en el sistema mundial. Barcelona /Madrid: Icaria/Fundación Hogar del Empleado, 1998, p. 43-74.

QUIJANO, Aníbal. Colonialidad del Poder y Clasificación Social. Journal of WorldSystems Research, v. 6, n. 2, p. 342-386, 2000.

QUIJANO, Aníbal; WALLERSTEIN, Immanuel. Americanity as a Concepto or the Americas in the Modern World System. International Journal of Social Sciences, Paris, n. 134, p. 549-559, 1992.

RITZER, George. Teoría sociológica contemporánea. Madrid: McGraw Hill, 1993.

STEFONI, Carolina. Panorama de la migración internacional en América del Sur. Serie Población y Desarrollo, n. 123. Santiago: CEPAL, 2018.

VARELA, Amarela. "Luchas migrantes": un nuevo campo de estudio para la sociología de los disensos. Andamios, v. 12, n. 28, p. 145-170, 2015.

VILLARREAL, María. Regionalismos e Migrações Internacionais na América do Sul. Contexto e perspectivas futuras sobre as experiências na CAN, no Mercosul e na Unasul. Revista Espaço Aberto, v. 8, n. 2, p. 131-148, 2018.

WALLERSTEIN, Immanuel. El moderno sistema mundial. La agricultura capitalista y los orígenes de la economía-mundo europea en el siglo XVI. México: Siglo XXI editores, 1979.

ZAVALA, María; PRIETO, Victoria. El papel de las migraciones en la población latinoamericana. Versión preliminar. In: GALEANA, Patricia (coord.) Historia comparada de las migraciones en las Américas. México, DF: Universidad Nacional Autónoma de México, 2014. 